\title{
Über die Wirkungsweise der Phosphatese.
}

\author{
II. Mitteilung.
}

Von

H. Euler und Hj. Ohlsén.

(Aus dem biochemischen Laboratorium der Hochschule Stockholm.)

(Der Redaktion zugegangen am 27. Dezember 1911.)

Unsere früheren Versuche über die enzymatische Phosphoresterbildung fortsetzend, haben wir die Frage, welches Kohlenhydrat der Veresterung unterliegt, von neuem einer eingehenden Prüfung unterzogen. Bei unseren bisher mitgeteilten Versuchen hatte sich gezeigt, daß reine Glukose durch Extrakt der Trockenhefe $\mathrm{H}$ der hiesigen St. Eriksbrauerei nicht oder fast nicht verestert wird, während die Veresterung mit Glukose, welche durch lebende Hefe vorbehandelt worden war, schnell und vollständig vonstatten geht.

Wir haben bereits früher betont, daß dieses Ergebnis nur dann erhalten wird, wenn gewisse, vermutlich nicht gärkräftige, Heferassen angewandt werden, daß also z. B. Münchener Hefe diesen Unterschied nicht oder weit schwächer erkennen läßt und zwar, wie leicht ersichtlich deshalb, weil durch die Trocknung dieser Hefen die Zymase extrahierbar wird.1) In diesen Fällen finden sich also im Extrakt der Trockenhefe diejenigen Gärungsenzyme, welche die Umwandlung der Glukose (bezw. der anderen Hexosen) bewirken.

1) Es sei ausdrücklich hervorgehoben, daß die erste Veröffentlichung über die Extrahierbarkeit der Zymase aus Hefe, welche bei Temperaturen von $20-35^{\circ}$ getrocknet ist, von A. v. Le be dew (Compt. rend., Bd. 152, S. 49, 1911) herrührt. Veranlaßt durch die Studien des hiesigen Laboratoriums über freie und gebundene Hefenenzyme (Vet. Ark. f. Kemi, Bd. 4, und Diese Zeitschrift, Bd. 73, S. 85, 1911) hat der eine von uns, unabhängig von Lebedew, Versuche über die Extrahierbarkeit der Zymase angestellt, welche aber erst in einer im Anfang Februar der hiesigen Akademie der Wiss, eingereichten, im April veröffentlichten Arbeit kurz erwähnt sind. Für unsere Versuche waren rein theoretische Gesichtspunkte maßgebend.

Die Angabe einer neuen Methode zur Gewinnung der *Zymase verdankt man ausschließlich Lebedew. 
Wie viel Zymase in Lösung geht bezw. extrahiert werden kann, ist bei allen Hefen stark von der Behandlung derselben abhängig, von der Dauer und Temperatur der Trocknung usw. Es ist deshalb von vornherein zu erwarten, daß in vielen Fällen auch aus gärschwachen Hefen gewisse, wenn auch geringe Mengen Zymase in den Extrakt übergehen und in diesen Fällen wird dann auch reine Glukose verestert, scheinbar direkt, tatsächlich aber nachdem andere Bestandteile der Zymase bereits auf die Hexose eingewirkt haben; von welcher Art diese Einwirkung ist, mag vorläufig außerhalb der Diskussion bleiben.1)

Wir gehen jetzt gleich zur Mitteilung unserer experimentellen Ergebnisse über.

Ein früherer Versuch hatte folgende Zahlen ergeben:

$25 \mathrm{ccm}$ Extrakt aus Trockenhefe

20 , $20 \%$ ige Glukoselösung

$10.5 \%$ ige $\mathrm{Na}_{2} \mathrm{HPO}_{4}$
$25 \mathrm{ccm}$ Extrakt aus Trockenhefe

20 , 20\% ige angegorene

Glukoselösung

$10,5 \%$ ige $\mathrm{Na}_{2} \mathrm{HPO}_{4}$

\begin{tabular}{c|c|c|c|c|c|c}
\hline Minuten . & 0 & 150 & 250 & 0 & 50 & 150 \\
\hline g $\mathrm{Mg}_{2} \mathrm{P}_{2} \mathrm{O}_{7} \cdot$ & 0,0398 & 0,0394 & 0,0394 & 0,0398 & 0,218 & 0,0000
\end{tabular}

Es muß besonders betont werden, daß die Vorbehandlung der Glukose, also die Herstellung der a angegorenen Glukose» mit lebender Hefe geschah. Von lebenden Hefezellen wurde die Glukoselösung durch sorgfältige Filtration befreit, die Lösung wurde durch etwa 10 Minuten langes Erhitzen sterilisiert. Der Extrakt der Trockenhefe wurde stets dargestellt durch Digerieren von etwa $50 \mathrm{~g}$ derselben mit der 5 fachen Menge Wasser während 3 Stunden bei $30^{\circ}$. Der Extrakt wurde gleich nach der Filtration zur Erhöhung seiner Wirksamkeit $\left.{ }^{2}\right)^{1 / 2}$ Stunde auf $40^{\circ}$ erhitzt.

Der reagierenden Mischung wurden von Zeit zu Zeit Proben entnommen, in welchen durch Zusatz von Ammoniak die Enzymwirkung gehemmt wurde.

1) Bezüglich der Rolle des Dioxyacetons "als Zwischenprodukt der Gärung verweisen wir auf die Notiz von H. Euler und S. Kullberg (Diese Zeitschr. Bd.76, S. 241, 1912) und tragen nach, daß Hr. v. Lebedew auch in Compt. rend. Bd. 153, S. 136, 1911 Versuche über die Veresterung des Dioxyacetons mitgeteilt hat.

2) Biochemische Zeitschrift, Bd. 37, S. 313, 1911. 
Die analytische Methode war die früher angewandte; die Proben wurden mit Magnesiamischung gefällt, und die Fällung wurde als $\mathrm{Mg}_{2} \mathrm{P}_{2} \mathrm{O}_{7}$ gewogen.

Die 5\% ige Lösung von $\mathrm{Na}_{2} \mathrm{HPO}_{4}$ wurde durch Abwägen des krystallisierten Salzes hergestellt: die genaue Konzentration wurde hierauf analytisch bestimmt.

$10 \mathrm{ccm}$ der $\mathrm{Na}_{2} \mathrm{HPO}_{4}$-Lösung wurden mit Wasser auf $55 \mathrm{ccm}$ verdünnt und dieser Lösung $10 \mathrm{ccm}$ entnommen. Dieselben enthielten:

$$
\begin{array}{ccc}
\text { Analyse } & 1 . & 0,0304 \mathrm{~g}^{2} \\
\mathrm{Mg}_{2} \mathrm{P}_{2} \mathrm{O}_{7} \\
\text { 2. } & \text { 2. } & 0,0305 * \mathrm{Mg}_{2} \mathrm{P}_{2} \mathrm{O}_{7} .
\end{array}
$$

In allen folgenden Versuchen enthält das Reaktionsgemisch von Anfang des Versuches mehr anorganisches Phosphat, als dem Gehalt der obigen Lösung entspricht. Dieser höhere Phosphatgehalt entstammt der Trockenhefe, wie folgender Versuch zeigt.

$50 \mathrm{~g}$ luftgetrocknete Trockenhefe $\mathrm{H}$ (Probe $15 \mathrm{HA}_{4}$ Öl) wurden im Thermostaten bei $30^{\circ}$ während 3 Stunden extrahiert.

$25 \mathrm{~g}$ Extrakt wurden mit $30 \mathrm{ccm}$ Wasser vermischt. Je $10 \mathrm{ccm}$ der Mischung wurden in der gewöhnlichen Weise mit Magnesiamischung gefällt und lieferten

$$
\begin{gathered}
\text { nach Analyse 1: } 0,0158 \mathrm{~g}^{\mathrm{Mg}_{2} \mathrm{P}_{2} \mathrm{O}_{7}} \\
\star \quad 2: 0,0157 \mathrm{Mg}_{2} \mathrm{P}_{2} \mathrm{O}_{7} .
\end{gathered}
$$

Andere Extrakte dürften noch mehr Phosphorsäure enthalten haben.

Diese Phosphorsäure entstammt den Nucleinsäuren der Hefe. Im Verlaufe der Reaktion wird diese Phosphorsäure durch die im Extrakt der Trockenhefe enthaltene Nuclease abgespalten und dann verestert. So kommt es, daß in den folgenden Versuchen I-V die Zahlen für $\mathrm{Mg}_{2} \mathrm{P}_{2} \mathrm{O}_{7}$ zu Anfang der Reaktion durchgehends höher sind als 0,0305 und nach einiger Zeit auf 0 heruntergehen.

\section{Versuch I.}

Hefe $\mathrm{H}$ im Vakuum zwischen $40^{\circ}$ und $50^{\circ}$ getrocknet. Im Versuch B wurde die Glukose mit lebender Hefe während etwa 10 Minuten angegoren, wobei die optische Drehung im $50 \mathrm{~mm}-\mathrm{Rohr}$ von $5,20^{\circ}$ auf $5,04^{\circ}$ zurückging; aus $150 \mathrm{ccm}$ Glukoselösung entwichen $35 \mathrm{ccm} \mathrm{CO}_{2}$. 


$$
\begin{aligned}
& 25 \mathrm{ccm} \text { Extrakt aus Trockenhefe }+ \\
& 10>5 \% \text { ige } \mathrm{Na}_{2} \mathrm{HPO}_{4}+
\end{aligned}
$$

A

\section{B}

$20 \mathrm{ccm}$ reine Glukoselösung $20 \mathrm{ccm}$ angegorene Glukoselösung

\begin{tabular}{c|c|c|c}
\hline \hline Minuten & g Mg$_{2} \mathrm{P}_{\mathbf{2}} \mathrm{O}_{7}$ & Minuten & g $\mathrm{Mg}_{\mathbf{2}} \mathrm{P}_{\mathbf{2}} \mathrm{O}_{\mathbf{7}}$ \\
\hline 0 & 0,0507 & 0 & 0,0500 \\
100 & 0,0500 & 100 & 0,0472 \\
200 & 0,0507 & 150 & 0,0450 \\
250 & 0,0502 & 250 & 0,0235
\end{tabular}

Der Versuch zeigt, wie die früheren, den großen Unterschied in der Reaktionsfähigkeit der reinen und der angegorenen Glukoselösung. Die erstere wurde überhaupt nicht verestert.

Mit der gleichen Hefe und der gleichen Mischung wurde ein Gärungsversuch angestellt, wobei die Mischung vorher mit $\mathrm{CO}_{2}$ gesättigt wurde. Eine $\mathrm{CO}_{8}$-Entwicklung war nicht meßbar.

\section{Versuch II $\alpha$.}

Hefe $\mathrm{H}$ im Vakuum bei $18^{\circ}$ während 7 Stunden getrocknet. Angegorene Glukoselösung: Während 10 Minuten wurden

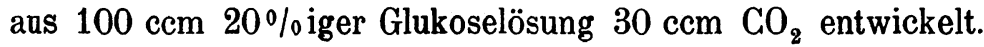
Drehungsrückgang: $5,33-5,170=3 \%$.

$25 \mathrm{ccm}$ Extrakt aus Trockenhefe +

\begin{tabular}{|c|c|c|c|c|c|}
\hline \multicolumn{2}{|c|}{$\begin{array}{c}\text { A } \\
20 \mathrm{ccm} \text { reine Glukose- } \\
\text { lösung }\end{array}$} & \multicolumn{2}{|c|}{$\begin{array}{c}\text { B } \\
20 \mathrm{ccm} \text { angegorene } \\
\text { Glukoselösung }\end{array}$} & \multicolumn{2}{|c|}{$\begin{array}{l}\text { C } \\
20 \mathrm{ccm} \text { angegorene, ge- } \\
\text { kockte Glukoselösung }\end{array}$} \\
\hline Minuten & g $\mathrm{Mg}_{2} \mathrm{P}_{2} \mathrm{O}_{7}$ & Minuten & g $\mathrm{Mg}_{2} \mathrm{P}_{2} \mathrm{O}_{7}$ & Minuten & $\mathrm{g} \mathrm{Mg}_{2} \mathrm{P}_{2} \mathrm{O}_{7}$ \\
\hline 0 & 0,0560 & 0 & 0,0560 & 0 & 0,0560 \\
\hline 62 & 0,0558 & 73 & 0,0541 & 67 & 0,0543 \\
\hline 130 & 0,0541 & 141 & 0,0504 & 135 & 0,0508 \\
\hline 361 & 0,0526 & 372 & 0,0317 & 366 & 0,0344 \\
\hline
\end{tabular}

$$
10,5 \% \text { ige } \mathrm{Na}_{2} \mathrm{HPO}_{4}+
$$

Ein Vergleich der Reihen B und C ergibt, daß die angegorene Glukoselösung durch Kochen nicht oder nur wenig verändert wird. Das aus der Glukose entstandene Produkt ist also jedenfalls wärmestabil. Die beiden Reaktionen $B$ und $C$ verliefen langsam, da die Glukoselösungen nach der Angärung 
und Filtration mit Thymol versetzt worden waren, wodurch eine Hemmung der Phosphatese eintrat.

Bei diesem Versuch war die Trocknung der Hefe in der Weise geschehen, daß der Extrakt offenbar etwas Zymase enthielt, so daß auch die nicht angegorene Glukose ein wenig verestert wurde.

\section{Versuch II $\beta$.}

Kommt eine gärkräftigere Hefe zur Anwendung, aus welcher nach v. Lebedew viel Zymase extrahiert werden kann, so wird bei obiger Versuchsanstellung natürlich auch Glukose verestert, welche nicht eigens vorher mit lebender Hefe vorbehandelt worden ist, da ja der Extrakt selbst Vergärung hervorruft.

«Münchener Hefe, nach v. Lebedew getrocknet» von Schroder in München. Extraktion während 2,5 Stunden bei $30^{\circ}$.

Angegorene Glukoselösung: Während 92 Minuten wurden aus $200 \mathrm{ccm} 20 \%$ iger Glukoselösung $620 \mathrm{ccm} \mathrm{CO}_{2}$ entwickelt. Drehungsrückgang $6,85-4,58^{\circ}$.

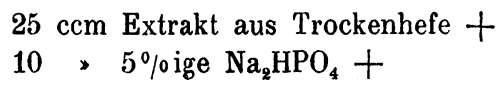

\begin{tabular}{c|c|c|c}
\multicolumn{2}{c|}{ A } & \multicolumn{2}{c}{$\mathrm{B}$} \\
\hline \hline Minuten & $\mathrm{g} \mathrm{Mg}_{2} \mathrm{P}_{2} \mathrm{O}_{7}$ & Minuten & $\mathrm{g} \mathrm{Mg}_{\mathbf{2}} \mathrm{P}_{\mathbf{2}} \mathrm{O}_{7}$ \\
\hline 0 & 0,0511 & 0 & 0,0532 \\
103 & 0,0510 & 118 & 0,0000 \\
274 & 0,0049 & 289 & 0,0000 \\
378 & 0,0000 & 393 & 0,0000
\end{tabular}

Gleichzeitig mit diesen Bestimmungen wurde ein Gärungsversuch angestellt, bei welchem die entweichende Kohlensäure gewogen wurde. Aus einer Lösung von der Zusammensetzung A (also $25 \mathrm{ccm}+10 \mathrm{ccm}$ Phosphatlösung $+20 \mathrm{ccm}$ reine Glukoselösung wurden $0,7223 \mathrm{~g} \mathrm{CO}_{2}$ während 372 Minuten entwickelt. 
Versuch III.

Die im Versuch II $\alpha$ erwähnte schädliche Einwirkung von Thymol kann durch folgende Messungen als festgestellt angesehen werden.

Hefe $\mathrm{H}$, im Vakuum bei $18^{\circ}$ während 6 Stunden getroeknet. Angegorene Glukoselösung. Während 10 Minuten werden aus $100 \mathrm{ccm} 20 \%$ iger Glukoselösung $26 \mathrm{ccm} \mathrm{CO} \mathrm{C}_{2}$ entwickelt: Drehungsrückgang $5,31-5,08^{\circ}$.

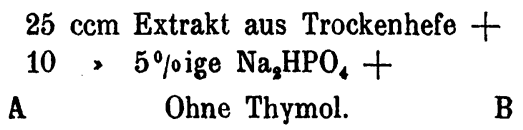
$20 \mathrm{ccm}$ angegorene Glukoselösung

\begin{tabular}{|c|c|c|c|}
\hline Minuten & g $\mathrm{Mg}_{2} \mathrm{P}_{2} \mathrm{O}_{7}$ & Minuten & g $\mathrm{Mg}_{8} \mathrm{P}_{8} \mathrm{O}_{7}$ \\
\hline 0 & 0,0500 & 0 & 0,0500 \\
\hline 100 & 0,0487 & 100 & 0,0450 \\
\hline 150 & 0,0480 & 150 & 0,0390 \\
\hline 200 & 0,0470 & 250 & 0,0240 \\
\hline
\end{tabular}

$20 \mathrm{ccm}$ reine Glukoselossung $\quad 20 \mathrm{ccm}$ angegorene Glukoselösung

\begin{tabular}{c|c|c|c}
\hline Minuten & $\mathrm{g} \mathrm{Mg}_{2} \mathrm{P}_{2} \mathrm{O}_{7}$ & Minuten & $\mathrm{g} \mathrm{Mg}_{2} \mathrm{P}_{\mathbf{2}} \mathrm{O}_{7}$ \\
\hline 0 & 0,0500 & 0 & 0,0500 \\
100 & 0,0490 & 100 & 0,0473 \\
150 & 0,0480 & 150 & 0,0455 \\
200 & 0,0475 & 250 & 0,0430
\end{tabular}

Ähnliche Resultate wurden auch mit anderen Bierhefen erhalten; so trat z. B. mit der früher von uns untersuchten Hamburger-Hefe eine fast vollständige Hemmung ein. Versuchsbedingungen wie oben angegeben.

Bierhefe der Hamburger Brauerei in Stockholm; getrocknet bei $18^{\circ}$.

Angegorene Glukose mit Thymol.

\begin{tabular}{c|c}
\hline Minuten & $\mathrm{g} \mathrm{Mg}_{2} \mathrm{P}_{2} \mathrm{O}_{7}$ \\
\hline 0 & 0,0570 \\
150 & 0,0543 \\
330 & 0,0484
\end{tabular}


Daß die Phosphatese von Thymol geschädigt wird, ist nicht besonders auffallend; Buchner hat bereits gefunden, daß diese Substanz die zellfreie Gärung hemmt, auch Amylasen werden durch Thymol inaktiviert.

Da nur der Zusatz eines wirksamen Antiseptikums die erforderliche Garantie dafür bietet, daß enzymatische Katalysen nicht durch die Tätigkeit lebender Zellen beeinflußt werden, haben wir dem Reaktionsgemisch stets Toluol zugesetzt, nachdem wir uns durch folgenden Versuch davon überzeugt hatten, daß ein solcher Zusatz die Wirkung der Phosphatese nicht beeinträchtigt.

\section{Versuch IV.}

Hefe H im Vakuum bei $18^{\circ}$ während 7 Stunden getrocknet. Angegorene Glukose; in 10 Minuten werden aus $100 \mathrm{ccm}$ $20 \%$ iger Glukoselösung $25 \mathrm{ccm} \mathrm{CO}_{2}$ entwickelt; Drehungsrückgang $5,27-5,05^{\circ}$.

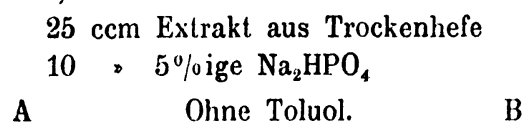

$20 \mathrm{ccm}$ reine Glukoselösung

$20 \mathrm{ccm}$ angegorene Glukoselösung

\begin{tabular}{c|c|c|c}
\hline Minuten & $\mathrm{g} \mathrm{Mg}_{8} \mathrm{P}_{2} \mathrm{O}_{7}$ & Minuten & $\mathrm{g} \mathrm{Mg}_{2} \mathrm{P}_{2} \mathrm{O}_{7}$ \\
\hline 0 & 0,0475 & 0 & 0,0475 \\
61 & 0,0458 & 66 & 0,0428 \\
282 & $0,0372 \quad 288$ & 0,0000 \\
& C & \multicolumn{2}{|c|}{ Mit Toluol. } \\
\end{tabular}

$20 \mathrm{ccm}$ reine Glukoselösung $20 \mathrm{ccm}$ angegorene Glukoselösung

\begin{tabular}{c|c|c|c}
\hline \hline Minuten & $\mathrm{g} \mathrm{Mg}_{2} \mathrm{P}_{\mathbf{z}} \mathrm{O}_{7}$ & Minuten & $\mathrm{g} \mathrm{Mg}_{2} \mathrm{P}_{\mathbf{2}} \mathrm{O}_{\overline{7}}$ \\
\hline 0 & 0,0473 & 0 & 0,0477 \\
49 & 0,0453 & 56 & 0,0428 \\
270 & 0,0357 & 276 & 0,0000
\end{tabular}

Toluol übt also keinen hemmenden Einfluß aus und bildet also für Versuche mit Phosphatese ein geeignetes Antiseptikum.

Die erste Mitteilung über Phosphatese enthält einen Versuch, nach welchem eine gewisse Menge Glukose um so un- 
vollständiger verestert wird, je weiter die vorherige Angärung fortgeschritten ist. (Tab. 9.) Da dieser Versuch für das Verständnis der Phosphatbindung nicht unwesentlich ist, so haben wir uns von der allgemeinen Gültigkeit unseres Ergebnisses nochmals überzeugen wollen und haben deswegen in folgendem Versuch sowohl die Konzentration des Phosphates als auch den Grad der Vergärung variiert.

\section{Versuch Va.}

Hefe $\mathrm{H}$ bei $18^{\circ}$ während 20 Stunden getrocknet.

Angegorene Glukoselösung A. In 64 Minuten werden

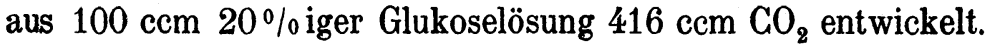

Drehungsrückgang $5,77-4,57^{\circ}$.

$25 \mathrm{ccm}$ Extrakt der Trockenhefe +

20 , angegorene Glukoselösung +

\begin{tabular}{c|c|c} 
I. & II. & III. \\
$10 \mathrm{ccm} 5 \%$ ige $\mathrm{Na}_{2} \mathrm{HPO}_{4}$ & $10 \mathrm{ccm} \mathrm{10 \%} \%$ ige $\mathrm{Na}_{2} \mathrm{HPO}_{4}$ & $15 \mathrm{ccm} \mathrm{10 \%} \%$ ige $\mathrm{Na}_{2} \mathrm{HPO}_{4}$ \\
\hline
\end{tabular}

\begin{tabular}{c|c|c|c|c|c}
\hline Minuten & $\mathrm{g} \mathrm{Mg}_{2} \mathrm{P}_{\mathbf{2}} \mathrm{O}_{7}$ & Minuten & $\mathrm{g} \mathrm{Mg}_{2} \mathrm{P}_{\mathbf{8}} \mathrm{O}_{7}$ & Minuten & $\mathrm{g} \mathrm{Mg}_{\mathbf{2}} \mathrm{P}_{8} \mathrm{O}_{7}$ \\
\hline 0 & 0,0444 & 0 & 0,0712 & 0 & 0,0909 \\
89 & 0,0303 & 79 & 0,0679 & 74 & 0,0900 \\
174 & 0,0000 & 164 & 0,0550 & 224 & 0,0881 \\
239 & 0,0000 & 230 & 0,0390 & 266 & 0,0855
\end{tabular}

Versuch Vb.

Hefe wie in Versuch Va.

Angegorene Glukoselösung B. In 106 Minuten werden aus $100 \mathrm{ccm} 20 \%$ iger Glukoselösung $880 \mathrm{~cm} \mathrm{CO} \mathrm{C}_{2}$ entwickelt. Drehungsrückgang $5,78-3,85^{\circ}$.

$25 \mathrm{ccm}$ Extrakt der Trockenhefe +

20 , angegorene Glukoselösung +

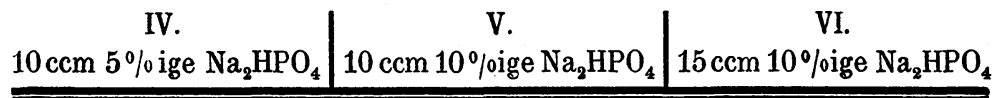

\begin{tabular}{c|c|c|c|c|c}
\hline \hline Minuten & $\mathrm{g} \mathrm{Mg}_{2} \mathrm{P}_{2} \mathrm{O}_{7}$ & Minuten & $\mathrm{g} \mathrm{Mg}_{2} \mathrm{P}_{2} \mathrm{O}_{7}$ & Minuten & $\mathrm{g} \mathrm{Mg}_{8} \mathrm{P}_{2} \mathrm{O}_{7}$ \\
\hline 0 & 0,0464 & 0 & 0,0732 & 0 & 0,0926 \\
74 & 0,0440 & 67 & - & 61 & 0,0905 \\
135 & 0,0386 & 128 & 0,0722 & 189 & - \\
201 & 0,0208 & 189 & 0,0709 & 231 & 0,0909 \\
\multicolumn{2}{l}{ Hoppe-Seyler's Zeitschrift f. physiol. Chemie. LXXXVI. } & & 30
\end{tabular}


Es sei zu den Versuchen $\mathrm{V}$ zunächst bemerkt, daß die Lösungen des Natriumphosphates stets aus Mono - und Dinatriumphosphat so hergestellt wurden, daß dieselben vollständig neutral gegen Lackmus waren. Es kann sich also bei den obigen Versuchen nicht ein verschiedener Einfluß der Alkalinität geltend gemacht haben.

Es ergibt sich also das auffallende Résultat, daß die Veresterungsgeschwindigkeit mit zunehmender Phosphatmenge abnimmt.

Ferner bestätigt sich unser früheres Ergebnis, daß die Phosphatbindung um so schneller verläuft, je weniger weit die Angärung fortgeschritten ist.

Da ein Überschuß von Phosphat hemmt, so war es nicht unwahrscheinlich, daß ein Zusatz von Ester die weitere Phosphatbindung ebenfalls beeinträchtigt. Folgender Versuch zeigt aber, daß dies nicht der Fall ist, vielmehr beschleunigt ein Zusatz eines Estersalzes die Phosphatbindung.

\section{Versuch VI.}

Hefe $\mathrm{H}$ bei $18^{0}$ getrocknet.

Angegorene Glukoselösung. Während 10 Minuten wurden $42 \mathrm{ccm} \mathrm{CO}_{2}$ entwickelt.

$15 \mathrm{ccm}$ Extrakt aus Trockenhefe +

$10 \curvearrowright 5 \%$ ige $\mathrm{Na}_{8} \mathrm{HPO}_{4}+$

20 angegorene Glukoselösung

\begin{tabular}{r|c|c|c|c|c}
\multicolumn{2}{c|}{ A. Ohne Zusatz. } & \multicolumn{2}{c}{ B. $+2,5$ g Esternatriumsalz. } \\
\hline \multirow{2}{*}{ Minuten } & \multicolumn{2}{|c|}{${\text { g } \mathrm{Mg}_{2} \mathrm{P}_{\mathbf{2}} \mathrm{O}_{7}}^{2}$} & Minuten & \multicolumn{2}{|c}{$\mathrm{g} \mathrm{Mg}_{2} \mathrm{P}_{\mathbf{2}} \mathrm{O}_{\mathbf{2}}$} \\
& I. & II. & & I. & II. \\
\hline 0 & 0,0460 & 0,0462 & 0 & 0,0460 & 0,0463 \\
103 & 0,0375 & 0,0393 & 95 & 0,0230 & 0,0239 \\
155 & 0,0205 & 0,0213 & 145 & 0,0107 & 0,0105 \\
242 & 0,0000 & 0,0000 & 232 & 0,0000 & 0,0000
\end{tabular}

Im Anschluß an die obigen Versuche sei in aller Kürze ein Resultat mitgeteilt, das der eine von uns gemeinschaftlich mit Herrn H. Bäckström gewonnen hat, und das uns für das Verständnis der physiologischen Wirkung des enzymatisch ge- 
bildeten Kohlenhydratphosphorsäureesters von Bedeutung zu sein scheint.

Das Natriumsalz des Kohlenhydratphosphorsäureesters wird zwar, wie Iwan off gefunden hat, durch Zymin, und wie Harden und Young gefunden haben, durch ein Enzym des Hefepreßsaftes, welches sie als Hexosenphosphatase bezeichnen, hydrolysiert. Lebende Hefe greift aber, wie ebenfalls durch Iwanoff und Harden und Young bekannt geworden ist, den Kohlenhydratphosphorsäureester bezw. dessen Salze nicht an. Nach S. G. Paine ${ }^{1}$ ) dringt auch nur ein kleiner Teil des Natriumsalzes aus einer Lösung in die Hefe ein.

Läßt man aber $20 \mathrm{ccm}$ einer 20\% igen Glukoselösung durch $0,25 \mathrm{~g}$ lebender Preßhefe vergären, so wird die Geschwindigkeit der Gärung durch Zusatz von 0,25 g des Natriumestersalzes verdoppelt. Das Salz selbst bleibt dabei so gut wie unverändert, höchstens Spuren davon werden gespalten; wieviel davon von der Hefe resorbiert wird, haben wir nicht untersucht. Diese Wirkung des Natriumestersalzes übertrifft diejenige des freien Natriumphosphates um etwa das Zehnfache. Dieses Resultat schließt sich an dasjenige von Iwan off (Zbl. f. Bakt., Bd. 24, S. 10, 1909) an, nach welchem die Triosophosphorsäure die Gärungswirkung des Hefanols stimuliert.

Das Natriumestersalz scheint hier also als Katalysator der Gärung durch lebende Hefe $z u$ fungieren. Dies würde für die Vermutung Iwan offs sprechen, daß das Salz des Kohlenhydratphosphorsäureesters bezw. des einen der beiden vermutlich existierenden, bei der Gärung auftretenden Ester mit dem von Harden und Young aufgefundenen und studierten "CoEnzym der Zymase» identisch ist. ${ }^{2}$ ) Jedoch ist zu bemerken, daß das "Co-Enzym» selbst die Gärung durch lebende Hefe noch viel stärker stimuliert als das Estersalz.

Leksand, Dezember 1911.

1) Proc. Roy. Soc. Biol., Vol. 84, p. 389, 1911.

$\left.{ }^{2}\right)$ Indessen muß daran erinnert werden, daß Hardens und Youngs Versuche (Zbl. f. Bakt., Bd. 26, S. 183, 1910) mit dieser Annahme nicht übereinstimmen. 